\title{
The role of the 'peer reviewer'
}

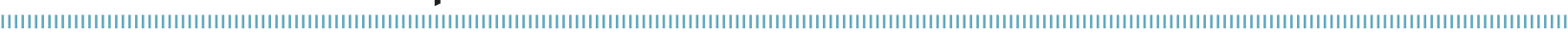

The method most commonly used by most of the Editorial Boards of scientific journals in article reviewing is a pre-evaluation made by the editors, followed by invitations to experts on the subject of that article. In most situations, the invited professionals have some work experience when conducting researches and publishing papers in good journals regularly. Unusually, young researchers without a lot of experience or tradition in publishing articles are invited as well, if showing expertise in reviewing manuscripts.

The pressure for more publications has required a lot from the Editorial Board when seeking professionals who are willing to make a good review and within a deadline. It's common for a journal editor to invite peer reviewers who do not accept a particular job, even if they are experts in the subject of the article. Several reasons are given to justify the rejection of article reviews, including: (i) excessive workload; (ii) the need to prepare reviews for other scientific journals or agencies; (iii) teaching work hours in the classroom (in the teachers' case); (iv) administrative functions etc.

Because of the difficulty in finding good peer reviewers, the Editorial Board is often forced to invite professionals whitout the proper experience and (sometimes) also without the skills to make a good evaluation of manuscripts. In many cases, the reviews are bad, and the editors are forced to discard some of them because of several mistakes. Here are listed some important points in the process of peer review of scientific articles:

1) Knowledge of the journal scope: it's important that the reviewer knows the purpose of the magazine; its main editorial lines; the reading audience; and the rigor with which the article must be evaluated.

2) Technical evaluation: reviewers should only accept to review an article if they feel familiar with the topic, as that's the only way they can actually evaluate the real contribution of the manuscript to the current state of art in the subject discussed.

3) Evaluating $\mathrm{x}$ suggesting: although many professionals can make excellent suggestions to the authors, this is not their main assignment. Many reviewers confuse peer reviewing with giving suggestions of corrections or including new sentences. Some even rewrite parts of the article. In general, what the editors require is a technical opinion on the quality of the article; its scientific basis; an evaluation if the authors actually clarify doubts/questions they intended to answer beforehand, based on their study; checking if the methods used are appropriate; checking interpretation/analysis of the results (if necessary) and the comparison to other bibliographic references. However, it's not rare to find peer reviewers who merely make suggestions to the authors, without dwelling on the quality of them. Still, it burdens the work of the Editor and adds little to the evaluation process of the manuscript.

4) Deadline: it's fundamental that reviewers respect the deadlines in order to avoid jeopardizing the whole process of manuscript evaluation. Unfortunately it's not rare to find professionals that do excellent reviews but never respect deadlines.

5) Cordiality: peer reviewers must point out corrections using a friendly vocabulary and, also, regarding the scientific aspect of the text. Instead of using sentences such as "The article is badly written and extremely simple", "The discussion of the results is bad", "The way of evaluating the results is totally wrong", "Many sentences are badly written and impossible to understand", "The hypotheses are badly formulated, what shows that the author has no idea of the subject", "The article is weak and does not add anything new to the field", "The results shown were obvious and they don't show anything new", "This article should be rejected", it's better to use sentences like "The article doesn't approach the subject with the proper profundity", "The discussion of the results is not adequate", "The way of evaluating the results was incorrect...", "Many sentences are confusing and difficult to understand", "The hypotheses are not clearly defined", "The actual contribution of the article was not very clear", "The results are only confirmatory and they can't justify the publication in this periodical".

As a conclusion, it is necessary a clear understanding of the major contribution of the peer reviewers in the manuscripts evaluation process and for the quality of the journals as well. Good reviewers are essential to increase the Editors' work efficiency. Thus, the Editorial Board should seek ways of rewarding good peer reviewers either with free copies of magazines or even by inviting them to join the Editorial Board, or so on. That way, there would be an additional motivation to those good peer reviewers who contribute to the quality of scientific journals, but rarely have their work duly acknowledged.

Luís Reynaldo Ferracciú Alleoni

Editor-in-Chief of the Scientia Agricola (www.scielo.br/sa) 